\title{
The Equivalent Inductance Silicon Micro-Pixel Avalance Photodiodes
}

\author{
E.A.Jafarova $^{1}$, Z.Y.Sadygov ${ }^{2}$, A.A.Dovlatov ${ }^{3}$, L.A.Aliyeva ${ }^{1}$, E.S.Tapdygov ${ }^{1}$, \\ K.A.Askerova ${ }^{1}$ \\ I'Institute of Physics Azerbaijan National Academy of Sciences Baku, AZ-1143, Azerbaijan, \\ ${ }^{2}$ (Joint Institute for Nuclear Research, 141980, Dubna, Russia, \\ ${ }^{3}$ (Department of Physics, Azerbaijan State Oil and Industry University, Baku, Azerbaijan,
}

\begin{abstract}
There have been investigated reactive properties of silicon avalanche photodiodes (MAPDMicropixel Avalanche Photodiode) with deeply buried micropixels (amplification channels) within AC signal frequencies $f=(50-500) \mathrm{kHz}$. By experiment is found out that measured capacitance of structures involving three $p$ - $n$ junctions in section passing through the pixels increases exponentially with $U_{\text {for }}$ (negative potential is applying to $n$-Si substrate) reaches maximum and at certain value $U_{f o r}=U_{i n v}$ changes the sign becoming the negative capacitance (equivalent inductance). The magnitude of active component of complete conduction $G$ grows with the applied voltage and reaches maximum value $\sim 70 \mathrm{mS}$ at $U_{f o r}=1,0 \mathrm{~V}(f=500 \mathrm{kHz})$. There has been calculated difference in phase $\varphi$ appearing between current and voltage and it is shown that at $U_{\text {for }}=0 \mathrm{~V}$ the $\varphi=$ $80^{\circ}$ and passes through the zero at $U_{\text {for }}=0,55 \mathrm{~V}$. The magnitude of negative capacitance recalculated to the inductance value with the growth of forward bias being decreased sharply tends to the saturation.
\end{abstract}

Keywords: micro-channel avalanche photodiode, $p$-n junction capacitance, $p$ - $n$ junction inductance, inversion voltage.

\section{INTRODUCTION}

In connection with the development of advanced technologies the creation of new high-technology, moderately priced photo-detectors with the high sensitivity and photon detection efficiency. For recent years elaborated micro-pixel avalanche photodiodes [1] have a high coefficient of internal multiplication $\left(\sim 10^{5}\right)$ and comparing with the existing vacuum photoelectric amplifiers (PEA) they are more compact, have high effective photodetection (30\%), no sensitive to the magnetic field effect, run at low operating voltages. MAPD have gained nide utilaziation at creating devices and equipment for scientific research, radiation control devices, medical research equipment and many fields of science and technology where ultrasensitive photo-detectors are needed. With various scintillation crystals MAPD can be used for making position and sensitive detectors of elementary particles and $\gamma$-radiation with high energy resolution.

MAPD construction involves pixel matrix of individual $\mathrm{n}^{+}$-regions of $2-5 \mu$ in diameter deeply buried into epitaxial layer of Si p-type conductivity grown on the surface of n-type Si substrate [1,2,4-7]. It allows the device high density of pixel for providing wide region of photoresponse linearity to be created [3]. For mentioned structures in the course of avalanche process the part of multiplied charge accumulates in potential well of pixel and runs down the substrate after passing main charge of avalanche. The process of charge accumulation in potential hole is the main process in the mechanism of negative feedback formation of this structure. It is necessary to note that the signal obtained from each pixel (Fig.1 region 4) after striking the light impulse on the photodiode surface is defined by the charge accumulated in each pixel. Therefore MAPD amplification factor one of the main parameters, is defined by the charge accumulated in the pixel capacitance $M=\frac{Q_{p x}}{q}$, where $Q_{p x}=C_{p x}\left(U_{r e v}-U_{b r}\right)$. Capacitance magnitude (equivalent inductance) also influences on such device parameters as quantum efficiency and noise factor.

Capacitance properties of micro-pixel avalanche photodiodes with deeply buried pixels have been investigated in [4,5,7]. It is shown that by applying inverse bias to the structure of three series connected $\mathrm{p}-\mathrm{n}$ junctions $n^{+}-p-n_{p x}{ }^{+} p$ (positive potential is applied to $n-S i$ substrate) the measured magnitude of total capacitance is defined as $1 / 2 C_{1}$, where $C_{1}$ is the capacitance of the first $n^{+}-p$ junction. It is necessary to note that the capacitance magnitude of the structures under the investigation and its change under the effect of external factors strongly influences on the recovery time parameter of these structures.

As it is noted in [1,3] MAPD structures run in the regime of avalanche multiplication of carriers when the positive potential is applied to $\mathrm{n}$-Si substrate. To enlarge the field of use of given devices and to reveal inertial process mechanism in them there have been investigated reaction properties of structures by applying negative potential to $\mathrm{n}-\mathrm{Si}$ substrate. 


\section{MEASUREMENT TECHNIQUE}

As a capacitometer there has been used the complete conductance bridge allowing the measurement by applying low alternating signal at frequencies $(50-500 \mathrm{kHz})$. Bridge supply is made out by $\mathrm{G} 7-112$ generator. As a zero indicator there has been used oscilloscope S1-70. As a preliminary the signal was amplified by selective micro-voltmeter VS-10. Stabilized supply power TEC-5010 provided DC feeds voltage across the sample and adjusted by potentiometer $\mathrm{R}$. Setup contains the capacitor $\mathrm{C}_{1}=10 \mu \mathrm{F}$ to provide of keeping measuring part out of the power unit interferences. Capacitance measurement accuracy is $\pm 5 \mathrm{pF}$. There has been also made thorough protection of samples against the light.

\section{PRODUCTION TECHNOLOGY}

Diodes under the investigation are made on $\mathrm{n}$-Si with $\rho$ - 7-10 Ohm.cm (Fig.1a). To improve field homogeneity on $\mathrm{n}-\mathrm{Si}$ (1) surface $\mathrm{n}^{+}$type thin layer (2) has been formed by ion doping. The choice of As ions instead of conventional $\mathrm{P}$ (phosphors) is related to its low diffusion rate at epitaxial layer deposition. Later the growth of the first epitaxial layer (3) of $4 \mu$ in thickness and $\rho=7 \mathrm{Ohm} \cdot \mathrm{cm}$ has been performed. By photolithography there has been formed the matrix of $\mathrm{n}^{+}$-region (4) through As ion doping. Then the second epitaxial layer of p-type (5) $4 \mu$ in thickness and $\rho=7 \mathrm{Ohm} \cdot \mathrm{cm}$ is grown. High-conducting layer of $\mathrm{p}^{+}$-type (6) has been created by $\mathrm{Br}$ ion doping on where aluminum contacts are deposited. Samples have the following geometric sizes: thickness of the first and second epitaxial layers of p-type conductivity (5) is $4 \mu$, specific resistance of both epitaxial layers is $7 \mathrm{Ohm} \cdot \mathrm{cm}$ (impurity concentration is $1.85 \cdot 10^{15} \mathrm{~cm}^{-3}$ ); diameter of pixels $\left(\mathrm{n}^{+}\right.$ - regions) is $2 \mu$ and thickness is about $\mathrm{r} \sim 0.8 \mu$; pixel surface density is $4 \cdot 10^{4} \mathrm{px} / \mathrm{mm}^{2}$. High conductively layer of p-type (6) with the impurity concentration $\mathrm{N}_{\mathrm{d}}>10^{18} \mathrm{~cm}^{-3}$ is set up by Brion-implantation doping Al contacts are deposited on it. The area of structures is $3 \times 3 \mathrm{~mm}^{2}$. Structures under the investigation are produced jointly with Zecotek Photonics Inc.

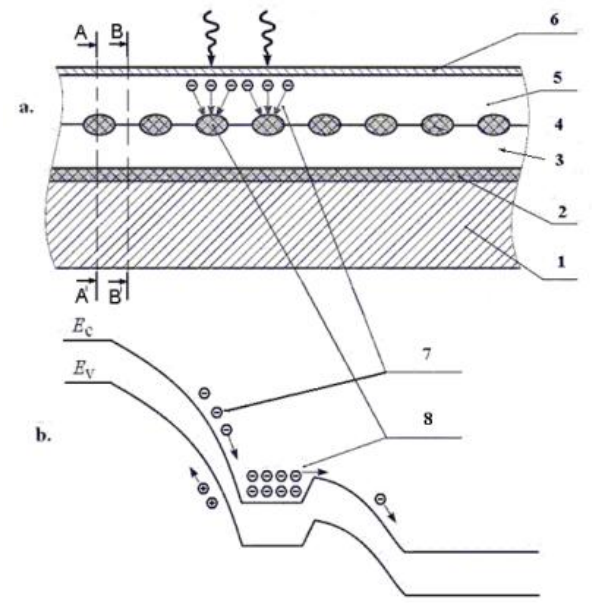

Fig.1 (a) - cross section MAPD-3N with deeply buried pixels; (b) - energy diagram through section A-A'; 7 - is the region of avalanche formation; 8 - is the potential well of forward-biased $\mathrm{p}-\mathrm{n}_{\mathrm{px}}{ }^{+}$junction.

Simulation impurity distribution in given structures and calculation results of impurity distribution in depth produced by above-mentioned technology of micro-pixel avalanche photodiodes are given in [6]. Thus by performing processing operations there have been created three $p-n$ junctions (section A-A' Fig. 1a): $n^{+}-p, p-$ $\mathrm{n}_{\mathrm{px}}{ }^{+}, \mathrm{n}_{\mathrm{px}}{ }^{+}-\mathrm{p}$ but in section B-B' not passing through regions $\mathrm{n}_{\mathrm{px}}{ }^{+}$there has been one $\mathrm{p}-\mathrm{n}$ junction on the substrate boundary with first epitaxial layer $\left(n^{+}-p\right)$. In Fig. 1 b the diagram of energy levels for A-A' section is given.

\section{RESULTS AND DISCUSSION}

As it mentioned in sections going through $\mathrm{n}^{+}$regions the structure under the investigation has three series-connected $\mathrm{p}-\mathrm{n}$ junctions $\mathrm{n}^{+}-\mathrm{p}, \mathrm{p}-\mathrm{n}_{\mathrm{px}}{ }^{+}, \mathrm{n}_{\mathrm{px}}{ }^{+}-\mathrm{p}$. In [4] it is shown that the observed dependence of MAPD$3 \mathrm{~N}$ photodiode capacitance of the frequency at small voltage $U_{\text {rev }}=0-3 \mathrm{~V}$ is related to the peculiarity of investigated structure. The presence of matrix of $n^{+}$regions between two epitaxial layers of p-type conductivity leads to the advent of effective resistance between layers. Influence of this resistance on magnitude of measured capacitance depends on the alternating signal frequency. By negative potential on $n-S i$ substrate the first $n^{+}-p$ junction biases in forward direction, but the middle $p-n^{+}$junction biases in reverse direction, therefore the first $\mathrm{n}^{+}$- $\mathrm{p}$ junction plays main role in physical processes going in structures under the study.

In Fig. 2 there has been presented the dependence $C\left(U_{f}\right)$ at three values of alternating signal frequency: 50,100 and $500 \mathrm{kHz}$. At small voltage $U_{\mathrm{f}}$ there has been observed the weak dependence of structure capacitance on the 
voltage and frequency, i.e. capacitance properties are due to the change of stationary acceptor concentration within the space charge of the first $n^{+}-p$ junction.

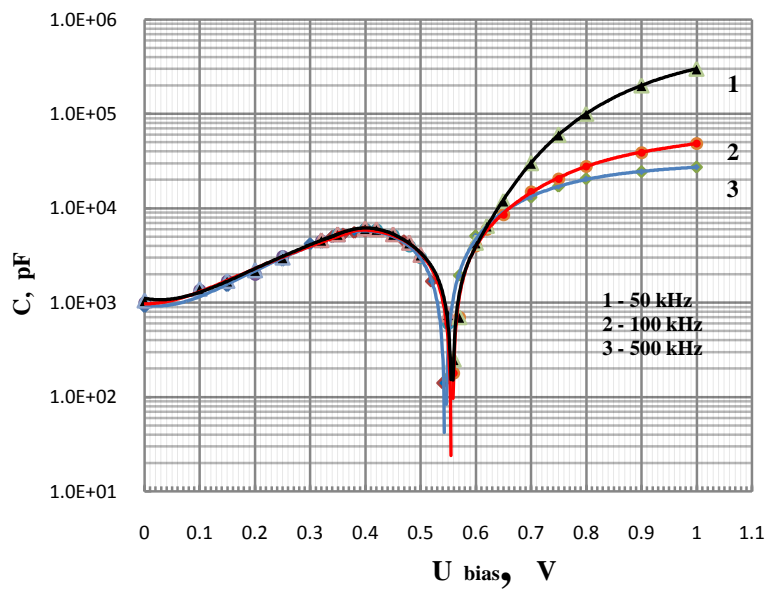

Fig.2. Dependence of micro-pixel avalanche photodiode (MAPD) capacitance on the voltage (negative potential is applied to $\mathrm{n}-\mathrm{Si})$ at three frequencies of alternating signal $(50 \mathrm{~Hz}, 100 \mathrm{~Hz}$ and $500 \mathrm{kHz})$.

Beginning with $U_{\mathrm{f}}=0.3 \mathrm{~V}$ the capacitance rises exponentially with the applied voltage:

$$
C_{f}=\text { const } \cdot \exp \frac{q U}{\beta_{c} k T}
$$

where $q$ is the electron charge, $k$ is the Boltzmann constant, coefficient $\beta_{c}$ takes on value $\beta_{c} \approx 3-3,5$.

On $C\left(U_{f}\right)$ curve at the certain value $U_{\max }<U_{\text {inv }}$ there has been observed maximum after which the measured capacitance decreasing down sharply to zero and goes into the negative capacitance (equivalent inductance). Voltage of inversion $U_{i n v}$ does not depend on the alternating signal frequency and within the experimental error changes over the range $(550 \pm 5) \mathrm{mV}$ by changing the frequency from 50 up to $500 \mathrm{kHz}$. Here for the convenience the negative capacitance $\mathrm{C}^{-}$is represented in the first quadrant.

As it is noted above even though MAPD structures under the investigation are made by epitaxial technique [5] the low of capacitance change with applied voltage is the same as for abrupt p-n junctions where unlike linear p-n junctions there is no dependence of inversion voltage on the frequency. At small voltage applied on the structure, when $U_{f}$ has order of diffusion potential difference as within the first $\mathrm{p}^{+}-\mathrm{n}$ junction as in near contact region of $\mathrm{Si}$ substrate the concentration of mobile carriers is low and reactance has a capacitance behavior. With the increasing of $U_{f}$ the concentration of mobile carriers within the space charge of the first p-n junction rises as much as that it can lead to the change of conductance in basic region. Base resistance modulation can be observed even at small currents yet insufficient for resistance change of all basic region but causing the slow increase of the conductance of its certain part. Changes of basic region conductance tends to exhibit its inertia and the reactance has an inductance behavior.

In paper [8] it is shown that impulse characteristics of voltage on the diode at big injection levels shows the voltage peak as it takes place when the current impulse passing through the inductance. Whereas at low injection levels observed distortion inherent to capacity. Active component of complete conductance G measured immediately on the bridge increases sharply with the applied voltage and reaches its maximum value $\sim 70 \mathrm{mS}$ at $U=1 \mathrm{~V}(\mathrm{f}=300 \mathrm{kHz})$.

Knowledge of capacitance magnitude $\mathrm{C}$ and conductance $\mathrm{G}$ also allows the phase shift between the current and voltage $\operatorname{tg} \varphi=\frac{\omega C}{G}$ to be calculated, where $\omega=2 \pi f$ is the angular frequency of alternating signal (Fig.3). As it is seen from the graph at $U_{f}<U_{i n v}$ the angle $\varphi$ is positive, i.e. reactance has a capacitance behavior but at $U_{f}>U$ ${ }_{i n v}, \varphi<0$ and reactance behaves as an inductance. In inversion point $U_{i n v}=0.55 \mathrm{~V}, \varphi=0$ and in this case MAPD structure resistance has a purely active behavior. 


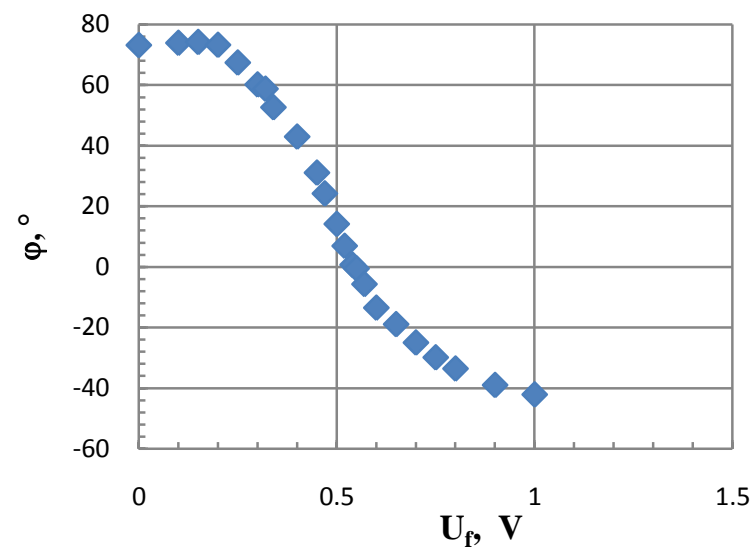

Fig.3 Dependence of phase shift angle between the current and voltage on the forward bias at $\mathrm{f}=500 \mathrm{kHz}$.

Density of the forward current in inversion point is approximately $2 \cdot 10^{-3} \mathrm{~A} / \mathrm{cm}^{2}$. Magnitude of equivalent inductance $L$ recalculated to magnitudes of negative capacitance $\mathrm{C}^{-}$for structures under study at two frequencies $(100 \mathrm{kHz}$ and $500 \mathrm{kHz})$ and at room temperature is given in fig. 4.

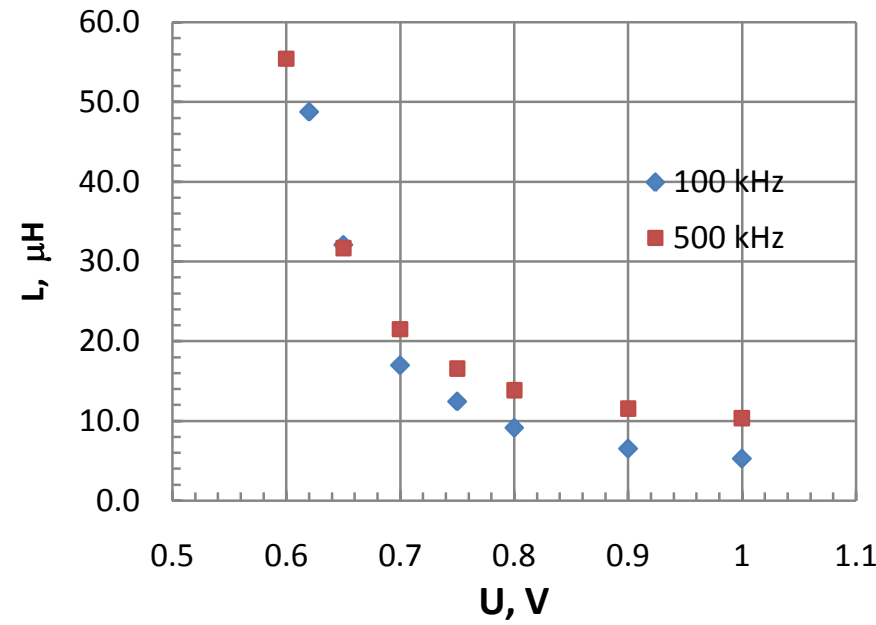

Fig. 4. Dependence of inductance magnitude on the forward bias at two alternating signal 100 and $500 \mathrm{kHz}$.

In this case the concentration of injected minority carriers $p_{n}^{i n j}$ varies by change of bias current from 0 up to $10 \mathrm{~mA}$ within $10^{6} \div 10^{15} \mathrm{~cm}^{-3}$. Capacitance inversion into equivalent inductance begins at $p_{n}^{i n j} \approx n_{n} \approx$ $7 \cdot 10^{14} \mathrm{~cm}^{-3}$, where $n_{n}$ is the concentration majority carriers in n-Si substrate. From Fig.4 it is seen that with the rise of forward bias $L$ being decreased sharply tends to the constant. Maximum value $L$ at $U_{\text {for }}$ is $\approx 50 \mu \mathrm{H}$. Given value is considerably more than the magnitude of film planar helix inductances is $\approx 15 \mu \mathrm{H}$ having a maximum diameter of helix no more than $10 \mathrm{~mm}$ and number of turns is no less than 100 used in integrated microelectronic circuits.

\section{CONCLUSION}

It is shown that silicon avalanche photodiodes with deeply buried pixels at certain value of voltage $U_{\text {for }}=U_{\text {inv }}$ may exhibit inductance properties. It is related to the change of initial n-Si substrate conductivity due to the considerable increase of mobile carrier concentration and charge accumulation within the first $n^{+}-p$ junction. Capacitance inversion into equivalent inductance begins at $p_{n}^{\text {inj }} \approx n_{n} \approx 7 \cdot 10^{14} \mathrm{~cm}^{-3}$. Inversion voltage of does not depend on the AC signal frequency $f=(50-500) \mathrm{kHz}$. Maximum magnitude of negative capacitance recalculated to the equivalent inductance value is $50 \mu \underline{\mathrm{H}}$ at $U_{\text {for }}=0.65 \mathrm{~V}(100 \mathrm{kHz})$. Thus investigated structures obtained by the technology used similarly at creating MAPD by fulfilling the condition $U_{\text {for }} \geq U_{\text {inv }}$ and $f \geq 50 \mathrm{kHz}$ have equivalent inductance which magnitude prevails considerably the inductance of semiconducting plane helixes $(15 \mu \mathrm{H})$ used in integrated microelectronic circuits. Maximum value of $L$ is $50 \mu \mathrm{H}$ at $U_{\text {for }}=0,65 \mathrm{~V}(f=100 \mathrm{kHz})$. 


\section{REFERENCES}

[1]. Z.Y. Sadygov. Micro-channel avalanche photodiode, Publish 10.02.2008, Patent №2316848 Russia, HOTL-31/06, Bull. №4.

[2]. Z.Y. Sadygov, F. Zerrouk, A.A. Dovlatov et. al., Performance of new micro-pixel avalanche photodiode from Zecotek Photonics, Nucl. Instr. Meth. A, 610, 2009, 381-383.

[3]. Z.Y. Sadygov, A.G. Olshevski, A.A. Dovlatov et.al. Micro-channel avalanche photodiode with rapid recovery time of parameters, Pisma v JTF, 39(11), 2013, 7-12.

[4]. E.A. Jafarova, Z. A. Iskenderzade, A.A. Dovlatov, E.S.Tapdygov, L.A. Alieva, Barrier capacitance of micro-pixel avalanche photodiodes. Transactions of Azerbaijan National Academy of Sciences, Physics and Astronomy, 37(2), 2013, 526-533.

[5]. E .A. Jafarova., Z.Y. Sadygov et. al., Barrier capacitance of micropixel avalanche photodiodes in different frequency, Proc.10 ${ }^{\text {th }}$ International Conference on Technical and Physical Problems of Electrical Engineering, Baku, Azerbaijan, 2014, 247-256.

[6]. Z.Y.Sadyqov, X.I.Abdullayev, F.I.Axmedov, E.A.Jafarova, A.A. Dovlatov, On features of potencial distribution in avalance photodiodes with deeply buried pixels. Fizika Sect.En., 19(2), 2013, 17-19.

[7]. E.A.Jafarova, Z.Y.Sadygov, F.I.Ahmadov, A.Z.Sadygov, A.A.Dovlatov, L.A.Aliyeva, E.S.Tapdyqov, On features of barrier capacitance of micropixel avalance photodiodes on different frequency, Theses of the $7^{\text {th }}$ Intern.Conf. on Materials and Condensed Matter Physics, Chisinau Moldova, 16-19 Sept.,2014, 266.

[8]. E.A.Jafarova, Deep impurity in germanium, silicon and nonstationary electronic processes in barrier structures on their basis, doctoral diss., Baku, 2007, 272p.

[9]. N.A. Poklonski, S.V. Shpakovski, N.I. Gorbachuk, S.B. Lastovski. Negative capacitance (inductive type impedance) of silicon $\mathrm{p}^{+}$-n junctions irradiated with fast electrons. Semiconductors, 40(7), 2006, 824-828. 